\title{
Lexical Disambiguation of Igbo through Diacritic Restoration
}

\author{
Ignatius Ezeani Mark Hepple Ikechukwu Onyenwe \\ NLP Research Group, \\ Department of Computer Science, \\ University of Sheffield, UK. \\ \{ignatius.ezeani,m.r.hepple, i.onyenwe\}@sheffield.ac.uk
}

\begin{abstract}
Properly written texts in Igbo, a low resource African language, are rich in both orthographic and tonal diacritics. Diacritics are essential in capturing the distinctions in pronunciation and meaning of words, as well as in lexical disambiguation. Unfortunately, most electronic texts in diacritic languages are written without diacritics. This makes diacritic restoration a necessary step in corpus building and language processing tasks for languages with diacritics. In our previous work, we built some $n$-gram models with simple smoothing techniques based on a closedworld assumption. However, as a classification task, diacritic restoration is well suited for and will be more generalisable with machine learning. This paper, therefore, presents a more standard approach to dealing with the task which involves the application of machine learning algorithms.
\end{abstract}

\section{Introduction}

Diacritics are marks placed over, under, or through a letter in some languages to indicate a different sound value from the same letter. English does not have diacritics (apart from a few borrowed words) but many of the world's languages use a wide range of diacritized letters in their orthography. Automatic Diacritic Restoration Systems (ADRS) enable the restoration of missing diacritics in texts. Many forms of such tools have been proposed, designed and developed but work on Igbo is still in its early stages.

\subsection{Diacritics and Igbo language}

Igbo, a major Nigerian language and the native language of the people of the south-eastern Nige- ria, is spoken by over 30 million people worldwide. It uses the Latin scripts and has many dialects. Most written works, however, use the official orthography produced by the Onwu Committe ${ }^{1}$.

The orthography has 8 vowels $(a, e, i, o, u, i, o$, $u$ ) and 28 consonants ( $b, g b, c h, d, f, g, g w, g h, h$, $j, k, k w, k p, l, m, n, n w, n y, \dot{n}, p, r, s, s h, t, v, w, y$, $z)$.

Table 1, shows Igbo characters with their orthographic or tonal (or both) diacritics and possible changes in meanings of the words they appear $\mathrm{in}^{2}$.

\begin{tabular}{|c|c|c|}
\hline Char & Ortho & Tonal \\
\hline$a$ & - & à,á, ā \\
\hline$e$ & - & è,é, è \\
\hline$i$ & ị & ì, í, $\overline{\mathrm{i}}, \grave{̣}, \underline{1}, \overline{\mathrm{i}}$ \\
\hline$o$ & o & ò, ó, ō, ọ̆, ọ, ọ \\
\hline$u$ & ụ & ù, ú, ū, ụ̂, ụ́, ̣̣ \\
\hline$m$ & - & m̀,ḿ, $\overline{\mathrm{m}}$ \\
\hline$n$ & $\dot{\mathrm{n}}$ & ǹ,ń, $\bar{n}$ \\
\hline
\end{tabular}

Table 1: Igbo diacritic complexity

Most Igbo electronic texts collected from social media platforms are riddled with flaws ranging from dialectal variations and spelling errors to lack of diacritics. For instance, consider this raw excerpt from a chat on a popular Nigerian online chat forum www.nairaland.com ${ }^{3}$ :

otu ubochi ka'm no na amaghi ihe mu na uwa ga-eje. kam noo n'eche ihe a,otu mmadu wee kpoturum, m lee anya o buru nwoke mara mma puru iche,mma ya turu m n'obi.o gwam si nne kedu

\footnotetext{
${ }^{1}$ http://www.columbia.edu/itc/mealac/pritchett/00fwp/igbo/ txt_onwu_1961.pdf

${ }^{2} m$ and $n$, nasal consonants, are sometimes treated as tone marked vowels.

${ }^{3}$ Source: http://www.nairaland.com/189374/igbo-lovemessages
} 
k'idi.onu ya dika onu ndi m'ozi, ihu ya dika anyanwu ututu,ahu ya n'achakwa bara bara ka mmiri si n'okwute. ka ihe niile no n'agbam n'obi,o sim na ohuru $m$ n'anya.na ochoro k'anyi buru enyi, a hukwuru m ya n'anya.anyi wee kwekorita wee buru enyi onye'a $m$ n'ekwu maka ya bu odinobi m,onye ihe ya n'amasi $m$

In the above example, you can observe that there is zero presence of diacritics - tonal or orthographic - in the entire text. As pointed out above, although there are other issues with regards to standard in the text, lack of diacritics seems to be harder to control or avoid than the others. This is partly because diacritics or lack of it does affect human understanding a great deal; and also the rigours a writer will go through to insert them may not worth the effort. The challenge, however, is that NLP systems built and trained with such poor quality non standard data will most likely be unreliable.

\subsection{Diacritic restoration and other NLP systems}

Diacritic restoration is important for other NLP systems such as speech recognition, text generation and machine translations systems. For example, although most translation systems are now very impressive, not a lot of them support Igbo language. However, for the few that do (e.g. Google Translate), diacritic restoration still plays a huge role in how well they perform. The example below shows the effect of diacritic marks on the output of Google Translate's Igbo-to-English translation.

\begin{tabular}{l|l|l}
\hline Statement & Google Translate & Comment \\
$\begin{array}{l}\text { O ji egbe ya gbuo egbe } \\
\text { O ji égbè ya gbuo égbé }\end{array}$ & $\begin{array}{l}\text { He used his gun to kill gun } \\
\text { He used his gun to kill kite }\end{array}$ & $\begin{array}{l}\text { wrong } \\
\text { correct }\end{array}$ \\
\hline \hline $\begin{array}{l}\text { Akwa ya di n'elu } a k w a \text { ya } \\
\text { Ákwà ya di n'elu àkwà ya }\end{array}$ & $\begin{array}{l}\text { It was on the bed in his room } \\
\text { his clothes on his bed }\end{array}$ & $\begin{array}{l}\text { fair } \\
\text { correct }\end{array}$ \\
\hline \hline $\begin{array}{l}\text { Oke riri } \text { oke ya } \\
\text { Òké riri òkè ya }\end{array}$ & $\begin{array}{l}\text { Her addiction } \\
\text { Mouse ate his share }\end{array}$ & $\begin{array}{l}\text { confused } \\
\text { correct }\end{array}$ \\
\hline \hline $\begin{array}{l}\text { O jiri } u g b o \text { ya bia } \\
\text { O jiri ụgbo ya bia }\end{array}$ & $\begin{array}{l}\text { He came with his farm } \\
\text { He came with his car }\end{array}$ & $\begin{array}{l}\text { wrong } \\
\text { correct }\end{array}$ \\
\hline
\end{tabular}

Table 2: Diacritic disambiguation for Google Translate

\subsection{Diacritic restoration and WSD}

Yarowsky (1994a) observed that, although diacritic restoration is not a hugely popular task in NLP research, it shares similar properties with such tasks as word sense disambiguation with regards to resolving both syntactic and semantic ambiguities. Indeed it was referred to as an instance of a closely related class of problems which includes word choice selection in machine translation, homograph and homophone disambiguation and capitalisation restoration (Yarowsky, 1994b).

Diacritic restoration, like sense disambiguation, is not an end in itself but an "intermediate task" (Wilks and Stevenson, 1996) which supports better understanding and representation of meanings in human-machine interactions. In most nondiacritic languages, sense disambiguation systems can directly support such tasks as machine translation, information retrieval, text processing, speech processing etc. (Ide and Véronis, 1998). But it takes more for diacritic languages, where possible, to produce standard texts. So for those languages, to achieve good results with such systems as listed above, diacritic restoration is required as a boost for the sense disambiguation task.

We note however, that although diacritic restoration is related to word sense disambiguation (WSD), it does not eliminate the need for sense disambiguation. For example, if the wordkey akwa is successfully restored to àkwà, it could still be referring to either bed or bridge. Another good example is the behaviour of Google Translate as the context around the word àkwà changes.

\begin{tabular}{l|l|l}
\hline Statement & Google Translate & Comment \\
\hline Akwa ya di n'elu $a k w a$ & It was on the high & confused \\
Akwa ya di n'elu $a k w a$ ya & It was on the bed in his room & fair \\
Ákwà ya di n'elu àkwà & His clothing was on the bridge & okay \\
Ákwà ya di n'elu àkwà ya & His clothing on his bed & good \\
\hline
\end{tabular}

Table 3: Disambiguation challenge for Google Translate

The last two statements, with proper diacritics on the ambiguous wordkey akwa seem both correct. Some disambiguation system in Google Translate must have been used to select the right form. However, it highlights the fact that such a disambiguation system may perform better when diacritics are restored.

\section{Problem Definition}

As explained above, lack of diacritics can often lead to some lexical ambiguities in written Igbo sentences. Although a human reader can, in most cases, infer the intended meaning from context, the machine may not. Consider the sentences in sections 2.1 and 2.2 and their literal translations: 
Input text:

Nwanyi ahu banyere n'ugbo ya.

Possible candidates:

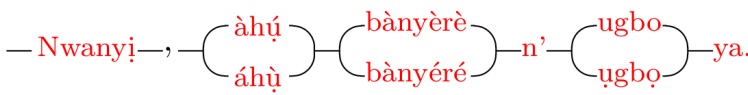

Most Probable Pattern:

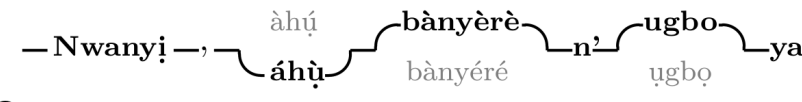

Output text:

Nwanyi áhụ bànyèrè n'ugbo ya.

Figure 1: Illustrative View of the Diacritic Restoration Process (Ezeani et al., 2016)

\subsection{Missing orthographic diacritics}

1. Nwanyi ahu banyere n'ugbo ya. (The woman entered her [farm|boat/craft])

2. O kwuru banyere olu ya. (He/she talked about his/her [neck/voice/work/job])

\subsection{Missing tonal diacritics}

1. Nwoke ahu nwere egbe n'ulo ya. (That man has a [gun $\mid$ kite] in his house)

2. O dina n'elu akwa. (He/she is lying on the [cloth $\mid$ bed,bridge $\mid$ egg $\mid$ cry] $)$

3. $\boldsymbol{E g} \boldsymbol{w} \boldsymbol{u}$ ji ya aka. (He/she is held/gripped by [fear|song/dance/music])

Ambiguities arise when diacritics - orthographic or tonal - are omitted in Igbo texts. In the first examples, we could see that ugbo(farm) and ugbog(boat/craft) as well as olu(neck/voice) and olu (work/job) were candidates in their sentences.

Also the second examples show that égbé(kite) and égbè(gun); ákwà(cloth), àkwà(bed or bridge), àkwá(egg), or even ákwá(cry) in a philosophical or artistic sense; as well as égwù(fear) and égwú(music) are all qualified to replace the ambiguous word in their respective sentences.

\section{Related Literature}

Diacritic restoration techniques for low resource languages adopt two main approaches: word based and character based.

\subsection{Word level diacritic restoration}

Different schemes of the word-based approach have been described. They generally involve preprocessing, candidate generation and disambiguation. Simard (1998) applied POS-tags and HMM language models for French. On the Croatian language, Šantić et al. (2009) used substitution schemes, a dictionary and language models in implementing a similar architecture. For Spanish, Yarowsky (1999) used dictionaries with decision lists, Bayesian classification and Viterbi decoding the surrounding context.

Crandall (2005), using Bayesian approach, HMM and a hybrid of both, as well as different evaluation method, attempted to improve on Yarowsky's work. Cocks and Keegan (2011) worked on Māori using naïve Bayes and wordbased $n$-grams relating to the target word as instance features. Tufiş and Chiţu (1999) used POS tagging to restore Romanian texts but backed off to character-based approach to deal with "unknown words". Generally, there seems to be a consensus on the superiority of the word-based approach for well resourced languages.

\subsection{Grapheme or letter level diacritic restoration}

For low-resource languages, there is often lack of adequate data and resources (large corpora, dictionaries, POS-taggers etc.). Mihalcea (2002) as well as Mihalcea and Nastase (2002) argued that letter-based approach will help to resolve the issue of lack of resources. They implemented instance based and decision tree classifiers which gave a high letter-level accuracy. However, their evaluation method implied a possibly much lower wordlevel accuracy.

Versions of Mihalcea's approach with improved evaluation methods have been implemented on other low resourced languages (Wagacha et al., 2006; De Pauw et al., 2011; Scannell, 2011). Wagacha et al. (2006), for example, reviewed the evaluation method in Mihalcea's work and introduced a word-level method for Gĩkũyũ. De Pauw et al. (2011) extended Wagacha's work by applying the method to multiple languages.

Our earlier work on Igbo diacritic restoration (Ezeani et al., 2016) was more of a proof of concept aimed at extending the initial work done by Scannell (2011). We built a number of $n$-gram models - basically unigrams, bigrams and trigrams - along with simple smoothing techniques. Although we got relatively high results, our evaluation method was based on a closed-world assumption where we trained and tested on the same set of data. Obviously, that assumption does not 
model the real world and so it is being addressed in this paper.

\subsection{Igbo Diacritic Restoration}

Igbo is low-resourced and is generally neglected in NLP research. However, an attempt at restoring Igbo diacritics was reported by Scannell (2011) in which a combination of word- and characterlevel models were applied. Two lexicon lookup methods were used: $L L$ which replaces ambiguous words with the most frequent word and $L L 2$ that uses a bigram model to determine the right replacement.

They reported word-level accuracies of $88.6 \%$ and $89.5 \%$ for the models respectively. But the size of training corpus ( $31 \mathrm{k}$ tokens with $4.3 \mathrm{k}$ word types) was too little to be representative and there was no language speaker in the team to validate the data used and the results produced. Therefore, we implemented a range of more complex $n$-gram models, using similar evaluation techniques, on a comparatively larger sized corpus $(1.07 \mathrm{~m}$ with $14.4 \mathrm{k}$ unique tokens) and had improved on their results (Ezeani et al., 2016).

In this work, we introduce machine learning approaches to further generalise the process and to better learn the intricate patterns in the data that will help better restoration.

\section{Experimental Setup}

\subsection{Experimental Data}

The corpus used in these experiments were collected from the Igbo version of the bible available from the Jehova Witness website ${ }^{4}$. The basic corpus statistics are presented in Table 4.

In Table 4, we refer to the "latinized" form of a word as its wordkey $^{5}$. Less than $10 \%(529 / 15696)$ of the wordkeys are ambiguous. However, these ambiguous wordkeys represent 529 ambiguous sets that yield 348,509 of the corpus words (i.e. words that share the same wordkey with at least one other word). These ambiguous words constitutes approximately $38.22 \%$ (348,509/911892) of the entire corpus. Some of the top most occurring, as well as the bottom least occurring ambiguous sets are shown in Table 5.

\footnotetext{
${ }^{4} \mathrm{jw}$. org

${ }^{5}$ Expectedly, many Igbo words are the same with their wordkeys
}

\begin{tabular}{l|r}
\hline Item & Number \\
\hline Total tokens & 1070429 \\
Total words & 902150 \\
Numbers/punctuations & 168279 \\
Unique words & 563383 \\
Ambiguous words & 348509 \\
Wordkeys & 15696 \\
Unique wordkeys & 15167 \\
Ambiguous wordkeys & 529 \\
$\quad$ 2 variants & 502 \\
3 variants & 15 \\
4 variants & 10 \\
5 variants & 2 \\
$\quad$ 5 variants & 0 \\
Approx. ambiguity & $38.22 \%$ \\
\hline
\end{tabular}

Table 4: Corpus statistics

\begin{tabular}{l|l}
\hline Top & Variants(count) \\
\hline na(29272) & ná(1332), na(27940) \\
$\mathrm{o}(22418)$ & $\mathrm{o}(4757)$, ò(64), ó(5), ọ(17592) \\
\hline Bottom & Variants(count) \\
\hline Giteyim(2) & Giteyịm(1), Giteyim(1) \\
Galim(2) & Galim(1), Galịm(1) \\
\hline
\end{tabular}

Table 5: Most and least frequent wordkeys

\subsection{Preprocessing}

The preprocessing task relied substantially on the approaches used by Onyenwe et al. (2014). Observed language based patterns were preserved. For example, $g a-, n a-$ and $n$ ' are retained as they are due to the specific lexical functions the special characters "-" or ", " confer on them. For instance, while $n a$ implies conjunction (e.g. ji $\boldsymbol{n a}$ ede: yam and cocoa-yam), $n a-$ is a verb auxiliary (e.g. Obi na-agba oso: Obi is running) and $n$ ' is shortened form of the preposition na (e.g. O di n'elu àkwà: It is on the bed.).

Also for consistency, diacritic formats are normalized using the unicode's Normalization Form Canonical NFC composition. For example, the character $e$ from the combined unicode characters $e$ (u0065) and ' (u0301) will be decomposed and recombined as a single canonically equivalent character $e^{\prime}(\mathrm{u} 00 \mathrm{e} 9)$. Also the character $\dot{n}$, which is often wrongly replaced with $\tilde{n}$ and $\bar{n}$ in some text, is generally restored back to its standard form.

The diacritic marking of the corpus used in this research is sufficient but not full or perfect. The orthographic diacritics (mostly dot-belows) have 
been included throughout. However, the tonal diacritics are fairly sparse, having been included only where they were for disambiguation (i.e. where the reader might not be able to decide the correct form from context.

Therefore, through manual inspection, some observed errors and anomalies were corrected by language speakers. For example, 3153 out of 3154 occurrences of the key mmadu were of the class mmadu. The only one that was mmadu was corrected to mmadu after inspection. By repeating this process, a lot of the generated ambiguous sets were resolved and removed from the list to reduce the noise. Examples are as shown in the table below:

\begin{tabular}{l|l|l|l}
\hline wordkey & Freq & var1Freq & var2Freq \\
\hline akpu & 106 & ákpụ-1 & akpụ-105 \\
agbu & 112 & agbụ-111 & ágbụ-1 \\
aka & 3690 & aka-3689 & ákà-1 \\
iri & 2036 & iri-2035 & ịịi-1 \\
\hline
\end{tabular}

Table 6: Some examples of corrected and removed ambiguous set

\subsection{Feature extraction for training instances}

The feature sets for the classification models were based on the works of Scannell (2011) on character-based restoration which was extended by Cocks and Keegan (2011) to deal with wordbased restoration for Māori. These features consist of a combination of $n$-grams - represented in the form $(x, y)$, where $x$ is the relative position to the target key and $y$ is the token length - at different positions within the left and right context of the target word. The datasets are built as described below for each of the ambiguous keys:

- FS1[(-1,1), (1,1)]: Unigrams on each side of the target key

- FS2[(-2,2), (2,2)]: Bigrams on each side

- FS3[(-3,3), (3,3)]: Trigrams on each side

- FS4[(-4,4), (4,4)]: 4-grams on each side

- FS5[(-5,5), (5,5)]: 5-grams on each side

- FS6[(-2,1), (-1,1), (1,1), (2,1)]: 2 unigrams on both sides

- FS7[(-3,1), $(-2,1),(-1,1),(1,1),(2,1),(3,1)]$ : 3 unigrams on each side
- FS8[(-4,1), $(-3,1),(-2,1),(-1,1),(1,1),(2,1)$, $(\mathbf{3 , 1}),(\mathbf{4 , 1})]$ : 4 unigrams on each side

- FS9[(-5,1), $(-4,1),(-3,1),(-2,1),(-1,1),(1,1)$, $(2,1),(3,1),(4,1),(5,1)]: 5$ unigrams on each side

- $\operatorname{FS10}[(-2,2),(-1,1),(1,1),(2,2)]: 1$ unigram and 1 bigram on each side

- FS11[(-3,3), $(-2,2),(2,2),(3,3)]$ : 1 bigram and 1 trigram on each side

- FS12[(-3,3), (-2,2), (-1,1), (1,1), (2,2), (3,3)]: 1 unigram, 1 bigram and 1 trigram on each side

- FS13](-4,4), $(-3,3),(-2,2),(-1,1),(1,1),(2,2)$, $(\mathbf{3 , 3}),(\mathbf{4 , 4 )})$ : 1 unigram, 1 bigram, 1 trigram and a 4 -gram on each side

\subsubsection{Appearance threshold and stratification}

We removed low-frequency wordkeys in our data by defining an appearance threshold as a percentage of the total tokens in our data. This is given by the

$$
\text { appThreshold }=\frac{C(\text { wordkeys })}{C(\text { tokens })} * 100
$$

and wordkeys with appThreshold below the stated value $^{6}$ were removed from the experiment.

As part of our data preparation for a standard cross-validation, we also passed each of our datasets through a simple stratification process. Instances of each label ${ }^{7}$, where possible, are evenly distributed to appear at least once in each fold or removed from the dataset.

Our stratification algorithm basically picks only labels from each dataset that have a population $p$ such that $p>=n$ folds. $n$ folds is the number of folds which in our case has a default value of 10 . In order to make the task a little more challenging, this process was augmented by the removal of some high frequency, but low entropy datasets where using the most common class (MCC) produces very high accuracies ${ }^{8}$. Entropy is loosely used here to refer to the degree of dominance of a particular class across the dataset and it is simply defined as:

$$
\text { entropy }=1-\frac{\max \left[\text { Count }_{\left(\text {label }_{i}\right)}\right]}{\text { len }(\text { dataset })}
$$

\footnotetext{
${ }^{6}$ In this work, we used an appThreshold of $0.005 \%$

${ }^{7}$ labels are basically diacritic variants.

${ }^{8}$ Datasets with more than $95 \%$ accuracy on the most common class (i.e. with entropy lower than 0.05 ) were removed.
} 
where $i=1 . . n$ and $n=$ number of distinct labels in the dataset. Table 7 shows 10 of the 30 lowest entropy datasets that were removed by this process.

\begin{tabular}{l|l|l|l} 
wordkey & Counts & MCCscore & Label(count) \\
\hline $\mathrm{e}(2)$ & 5476 & $99.78 \%$ & è(12); $\mathrm{e}(5464)$ \\
anyi(2) & 5390 & $99.63 \%$ & anyị(5370); ànyị(20) \\
ma(2) & 6713 & $99.61 \%$ & ma(6687); mà(26) \\
ike(2) & 3244 & $99.54 \%$ & ikè(15); ike(3229) \\
unu(2) & 8662 & $99.53 \%$ & ùnu(41); unu(8621) \\
$\mathrm{Ha}(2)$ & 2266 & $99.29 \%$ & Hà(16); Ha(2250) \\
$\mathrm{a}(2)$ & 12275 & $99.10 \%$ & $\mathrm{a}(12165) ;$ à(110) \\
onye(2) & 8937 & $98.87 \%$ & onye(8836); ònye(101) \\
ohu(2) & 790 & $98.73 \%$ & ohu(780); ohụ(10) \\
eze(2) & 2633 & $98.14 \%$ & eze(2584); ezé(49) \\
\hline
\end{tabular}

Table 7: Low entropy datasets

At end of these pruning processes, our remaining datasets came to 110 with the distribution as follows:

- datasets with only 2 variants: $=93$

- datasets with 3 variants: $=7$

- datasets with 4 variants: $=8$

- datasets with 5 variants: $=2$

Some datasets that originally had multiple variants lost some of their variants. For example, the dataset from akwa which originally had five variants and 1067 instances comprising of ákwá (355), ákwà(485), akwa(216), àkwà(1) and àkwá(10) retained only four variants (after dropping àkwà) and 1066 instances.

\subsection{Classification algorithms}

This work applied versions of five of the commonly used machine learning algorithms in NLP classification tasks namely:

- Linear Discriminant Analysis(LDA)

- K Nearest Neighbors(KNN)

- Decision Trees(DTC)

- Support Vector Machines(SVC)

- Naïve Bayes(MNB)

Their default parameters on Scikit-learn toolkit were used with 10-fold cross-validation and the evaluation metrics used is mainly the accuracy of prediction of the correct diacritic form in the test data. The effect of the accuracy obtained for a

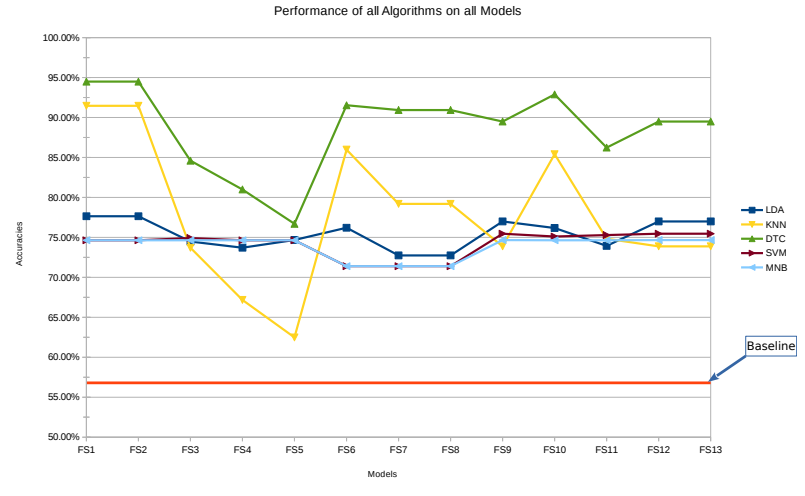

Figure 2: Evaluation of algorithm performance on each feature set model

dataset on the overall performance depends on the weight of the dataset. Each dataset is assigned a weight corresponding to the number of instances it generates from the corpus which is determined by its frequency of occurrence.

So the actual performance of each learning algorithm, on a particular feature set model, is the overall weighted average of the its performances across all the 110 datasets. The bottom line accuracy is the result of replacing each word with its wordkey which gave an accuracy of $30.46 \%$. However, the actual baseline to beat is $\mathbf{5 2 . 7 9 \%}$ which is achieved by always predicting the most common class.

\subsection{Results and Discussions}

The results of our experiments are as shown in Table 8 and Figure 2.

\begin{tabular}{l|l|l|l|l|l}
\hline Models & LDA & KNN & DTC & SVM & MNB \\
\hline \hline Baseline: & $\mathbf{5 2 . 7 9 \%}$ & \multicolumn{5}{|l}{} \\
\hline FS1 & $\mathbf{7 7 . 6 5 \%}$ & $\mathbf{9 1 . 4 7 \%}$ & $\mathbf{9 4 . 4 9} \%$ & $74.64 \%$ & $74.64 \%$ \\
FS2 & $\mathbf{7 7 . 6 5 \%}$ & $\mathbf{9 1 . 4 7 \%}$ & $\mathbf{9 4 . 4 9} \%$ & $74.64 \%$ & $74.64 \%$ \\
FS3 & $74.48 \%$ & $73.70 \%$ & $84.60 \%$ & $74.92 \%$ & $74.64 \%$ \\
FS4 & $73.71 \%$ & $67.18 \%$ & $81.00 \%$ & $74.64 \%$ & $74.64 \%$ \\
FS5 & $74.68 \%$ & $62.48 \%$ & $76.70 \%$ & $74.64 \%$ & $74.64 \%$ \\
FS6 & $76.21 \%$ & $85.98 \%$ & $91.54 \%$ & $71.39 \%$ & $71.39 \%$ \\
FS7 & $72.74 \%$ & $79.20 \%$ & $90.94 \%$ & $71.39 \%$ & $71.39 \%$ \\
FS8 & $72.74 \%$ & $79.20 \%$ & $90.94 \%$ & $71.39 \%$ & $71.39 \%$ \\
FS9 & $76.99 \%$ & $73.88 \%$ & $89.50 \%$ & $\mathbf{7 5 . 4 6 \%}$ & $\mathbf{7 4 . 6 7 \%}$ \\
FS10 & $76.18 \%$ & $85.41 \%$ & $92.89 \%$ & $75.11 \%$ & $74.64 \%$ \\
FS11 & $73.94 \%$ & $74.83 \%$ & $86.23 \%$ & $\mathbf{7 5 . 2 9 \%}$ & $\mathbf{7 4 . 6 4 \%}$ \\
FS12 & $76.99 \%$ & $73.88 \%$ & $89.50 \%$ & $\mathbf{7 5 . 4 6 \%}$ & $\mathbf{7 4 . 6 7 \%}$ \\
FS13 & $76.99 \%$ & $73.88 \%$ & $89.50 \%$ & $\mathbf{7 5 . 4 6 \%}$ & $\mathbf{7 4 . 6 7 \%}$ \\
\hline
\end{tabular}

Table 8: Summary of results

The experiments indicate that on the average all the algorithms were able to beat the baseline on all models. The decision tree algorithm (DTC) performed best across all models with an average accuracy of $88.64 \%$ (Figure 3), and the highest accuracy of $94.49 \%$ (Table 8 ) on both the FS1 and FS2 
models. However, with an average standard deviation of 0.076 (Figure 4) for its results, it appears to be the least reliable.

As the next best performing algorithm, KNN falls below DTC in average accuracy (91.47\%) but seems slightly more reliable. It did, however, struggle more than others as the dimension of feature $n$-grams increased (see its performance on FS3, FS4 and FS5). This may be due to the increase in sparsity of features and the difficulty to find similar neighbours. The other algorithms LDA, SVM and MNB - just trailed behind and although their results are a lot more reliable especially SVM and MNB (Figure 4). But this may be an indication that their strategies are not explorative enough. However, it could be observed that they traced a similar path in the graph and also had their highest results with the same set of models (i.e. FS9, FS12 and FS13) with wider context.

On the models, we observed that the unigrams and bigrams have better predictive capacity than the other $n$-grams. Most of the algorithms got comparatively good results with FS1, FS2, FS6 and FS10 (Figure 5) each of which has the unigram closest to the target word (i.e. in the \pm 1 position) in the feature set. Also, models that excluded the closest unigrams on both sides (e.g. FS11) and those with fairly wider context did not perform comparatively well across algorithms.

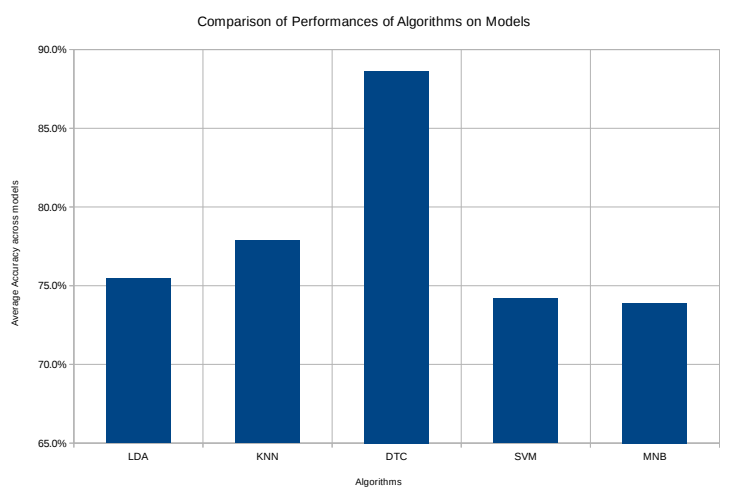

Figure 3: Average performance of algorithms

Again, it appears that beyond the three closest unigrams (i.e. those in the -3 through +3 positions), the classifiers tend to be confused by additional context information. Generally, FS1 and FS2 stood out across all algorithms as the best models while FS6 and FS7 also did well especially with DTC, KNN and LDA.

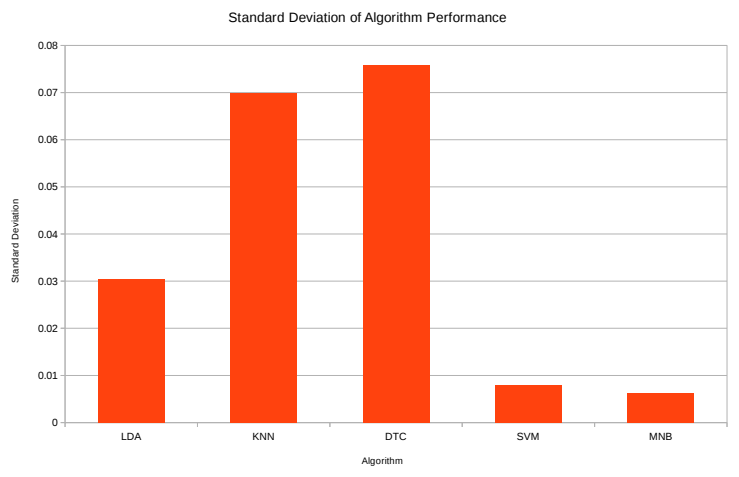

Figure 4: Average standard deviation for algorithms

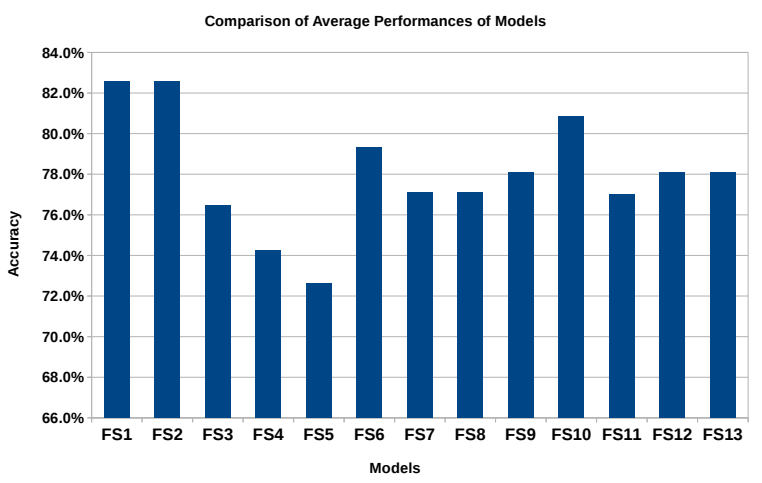

Figure 5: Average performance of models

\subsection{Future Research Direction}

Although our results show a substantial improvement from the baseline accuracy by all the algorithms on all the models, there is still a lot of room for improvement. Our next experiments will involve attempts to improve the results by focusing on the following key aspects:

- Reviewing the feature set models:

So far we have used instances with similar features on both sides of the target words. In our next experiments, we may consider varying these features.

\section{- Exploiting the algorithms:}

We were more explorative with the algorithms and so only the default parameters of the algorithms on Scikit-learn were tested. Subsequent experiments will involve tuning the parameters of the algorithms and possibly using more evaluation metrics.

\section{- Expanding data size and genre:}

A major challenge for this research work is lack of substantially marked corpora. So although, we achieved a lot with the bible data, 
it is inadequate and not very representative of the contemporary use of the language. Future research efforts will apply more resources to increasing the data size across other genres.

\section{- Predicting unknown words:}

Our work is yet to properly address the problem of unknown words. We are considering a closer inspection of the structural patterns in the target word to see if they contain elements with predictive capacity.

\section{- Broad based standardization:}

Beside lack of diacritics online Igbo texts are riddled with spelling errors, lack of standard orthographic and dialectal forms, poor writing styles, foreign words and so on. It may therefore be good to consider a broader based process that includes, not just diacritic restoration but other aspects of standardization.

- Interfacing with other NLP systems:

Although it seems obvious, it will be interesting to investigate, in empirical terms, the relationship between diacritic restoration and others NLP tasks and systems such as POStagging, morphological analysis and even the broader field of word sense disambiguation.

\section{Acknowledgements}

The IgboNLP Project @ The University of Sheffield, UK is funded by TETFund \& Nnamdi Azikiwe University, Nigeria.

\section{References}

John Cocks and Te-Taka Keegan. 2011. A Wordbased Approach for Diacritic Restoration in Māori. In Proceedings of the Australasian Language Technology Association Workshop 2011, pages 126-130, Canberra, Australia, December.

David Crandall. 2005. Automatic Accent Restoration in Spanish text. http://www.cs.indiana.edu/ djcran/projects/ 674_final.pdf. [Online: accessed 7-January-2016].

Guy De Pauw, Gilles maurice De Schryver, Laurette Pretorius, and Lori Levin. 2011. Introduction to the Special Issue on African Language Technology. Language Resources and Evaluation, 45:263-269.

Ignatius Ezeani, Mark Hepple, and Ikechukwu Onyenwe, 2016. Automatic Restoration of Diacritics for Igbo Language, pages 198-205. Springer International Publishing, Cham.
Nancy Ide and Jean Véronis. 1998. Introduction to the special issue on word sense disambiguation: The state of the art. Comput. Linguist., 24(1):2-40, March.

Rada F. Mihalcea and Vivi Nastase. 2002. Letter level learning for language independent diacritics restoration. In: Proceedings of CoNLL-2002, Taipei, Taiwan, pages 105-111.

Rada F. Mihalcea. 2002. Diacritics Restoration: Learning from Letters versus Learning from Words. In: Gelbukh, A. (ed.) CICLing LNCS, pages 339348 .

Ikechukwu Onyenwe, Chinedu Uchechukwu, and Mark Hepple. 2014. Part-of-speech Tagset and Corpus Development for igbo, an African Language. LAW VIII - The 8th Linguistic Annotation Workshop., pages 93-98.

Kevin P. Scannell. 2011. Statistical unicodification of african languages. Language Resource Evaluation, 45(3):375-386, September.

Michel Simard. 1998. Automatic Insertion of Accents in French texts. Proceedings of the Third Conference on Empirical Methods in Natural Language Processing, pages 27-35.

Dan Tufiş and Adrian Chiţu. 1999. Automatic Diacritics Insertion in Romanian texts. In Proceedings of COMPLEX99 International Conference on Computational Lexicography, pages 185-194, Pecs, Hungary.

Nikola Šantić, Jan Šnajder, and Bojana Dalbelo Bašić, 2009. Automatic Diacritics Restoration in Croatian Texts, pages 126-130. Dept of Info Sci, Faculty of Humanities and Social Sciences, University of Zagreb , 2009. ISBN: 978-953-175-355-5.

Peter W. Wagacha, Guy De Pauw, and Pauline W. Githinji. 2006. A Grapheme-based Approach to Accent Restoration in Gikũyũ. In Proceedings of the Fifth International Conference on Language Resources and Evaluation, pages 1937-1940, Genoa, Italy, May.

Yorick Wilks and Mark Stevenson. 1996. The grammar of sense: Is word-sense tagging much more than part-of-speech tagging? CoRR, cmp-lg/9607028.

David Yarowsky. 1994a. A Comparison of Corpusbased Techniques for Restoring Accents in Spanish and French Text. In Proceedings, 2nd Annual Workshop on Very Large Corpora, pages 19-32, Kyoto.

David Yarowsky. 1994b. Decision Lists for Lexical Ambiguity Resolution: Application to Accent Resolution in Spanish and French Text. In Proceedings of ACL-94, Las Cruces, New Mexico.

David Yarowsky, 1999. Corpus-based Techniques for Restoring Accents in Spanish and French Text, pages 99-120. Kluwer Academic Publishers. 